BASIC RESEARCH

\title{
Phagocytic responses of peritoneal macrophages and neutrophils are different in rats following prolonged exercise
}

\author{
Clílton K. O. Ferreira,' Jonato Prestes," Felipe F. Donatto,' Rozangela Verlengia,' James W. Navalta,"' \\ Cláudia R. Cavaglieri' \\ 'Health Science Faculty, Methodist University of Piracicaba, São Paulo, Brazil. "Graduate Program of Physical Education and Health, Catholic University of \\ Brasilia, Brasilia, Brazil. "'Department of Kinesiology, Western Kentucky University, Bowling Green, Kentucky.
}

OBJECTIVE: To analyze the effects of exhausting long-duration physical exercise (swimming) sessions of different durations and intensities on the number and phagocytic capacity of macrophages and neutrophils in sedentary rats.

INTRODUCTION: Exercise intensity, duration and frequency are important factors in determining immune response to physical effort. Thus, the effects of exhausting long-duration exercise are unclear.

METHODS: Wistar rats were divided into two groups: an untreated group (macrophage study) and oyster glycogentreated rats (neutrophil study). In each group, the animals were subdivided into five groups (10 rats per group): unexercised controls, an unadapted low-intensity exercise group, an unadapted moderate-intensity exercise group, a preadapted low-intensity exercise group and a preadapted moderate-intensity exercise group. All exercises were performed to exhaustion, and preadaptation consisted of 5, 15, 30 and 45 min sessions.

RESULTS: Macrophage study: the number of peritoneal macrophages significantly decreased $\left(9.22 \pm 1.78 \times 10^{6}\right)$ after unadapted exercise but increased $\left(21.50 \pm 0.63 \times 10^{6}\right)$ after preadapted low-intensity exercise, with no changes in the moderate-intensity exercise group. Phagocytic capacity, however, increased by more than $80 \%$ in all exercise groups (low/moderate, unadapted/preadapted). Neutrophil study: the number of peritoneal neutrophils significantly decreased after unadapted $\left(29.20 \pm 3.34 \times 10^{6}\right)$ and preadapted $\left(50.00 \pm 3.53 \times 10^{6}\right)$ low-intensity exercise but increased after unadapted $\left(127.60 \pm 5.14 \times 10^{6}\right)$ and preadapted $\left(221.80 \pm 14.85 \times 10^{6}\right)$ moderate exercise. Neutrophil phagocytic capacity decreased by $63 \%$ after unadapted moderate exercise but increased by $90 \%$ after corresponding preadapted sessions, with no changes in the low-intensity exercise groups.

CONCLUSION: Neutrophils and macrophages of sedentary rats respond differently to exercise-induced stress. Adaptation sessions reduce exercise-induced stress on the immune system.

KEYWORDS: Exercise; Immune System; Neutrophils; Macrophages; Phagocytic Capacity.

Ferreira CKO, Prestes J, Donatto FF, Verlengia R, Navalta JW, Cavaglieri CR. Phagocytic responses of peritoneal macrophages and neutrophils are different in rats following prolonged exercise. Clinics. 2010;65(11):1167-1173.

Received for publication on June 24, 2010; First review completed on July 10, 2010; Accepted for publication on August 10, 2010

E-mail: ccavagli@unimep.br

Tel.: 5519 3124-1515

\section{INTRODUCTION}

It has been shown that stress induced by exercise stimulates the phagocytic capacity of macrophages and neutrophils. ${ }^{1}$ As a result, the general stimulation of phagocytosis during exhausting physical exercise can potentially compensate for a decrease in lymphoid activity, thus preventing the entrance and survival of microorganisms in situations of immunodepression. ${ }^{2}$ In some cases, this response is also mediated by stress hormones, such as

Copyright (c) 2010 CLINICS - This is an Open Access article distributed under the terms of the Creative Commons Attribution Non-Commercial License (http:// creativecommons.org/licenses/by-nc/3.0/) which permits unrestricted noncommercial use, distribution, and reproduction in any medium, provided the original work is properly cited. glucocorticoids, which are immunosuppressive in lymphocytes. $^{3}$

Glucocorticoids can exert different effects on nonspecific functions of macrophages, including chemotaxis and phagocytosis, as well as more specific functions such as the presentation of antigens. ${ }^{4,5}$ Neutrophils and monocytes can be stimulated by catecholamines. ${ }^{1,6,7}$ Phagocytosis and catecholamine levels have been proposed to be neuroimmune-endocrine physiological markers in athletes. ${ }^{8}$ Other hormones, such as thyroid hormones (T3 and T4), prolactin (PRL), growth hormones (GH) and B-endorphins, contribute in general to the effects of exercise-induced stress on phagocytosis. , $^{9} 10$

Although several investigations have demonstrated a correlation between hormone or neuropeptide levels and the immune response to acute exercise, few studies have 
attempted to evaluate the phagocytic response to an adaptation period of exercise. ${ }^{11}$ Batista et al. ${ }^{12}$ stated that reports on the function of peritoneal macrophages following exercise training are rather controversial. For example, Vitorino et al. ${ }^{13}$ found no effect of shark liver oil supplementation, exercise or their combination on the innate immune system. ${ }^{13}$ This response depends on variables of physical exercise, such as intensity, duration and frequency, along with the exercise type. In addition, few studies have evaluated the immunomodulatory effect of exercise training on peritoneal macrophage function, as activated macrophages are important sources of proinflammatory factors.

Several studies, however, have shown that regular moderate exercise stimulates many aspects of the immune response. ${ }^{11,14,15}$ Dos Santos et al. ${ }^{16}$ showed that the increase in macrophage function induced by exercise is supported by enhanced glutamine consumption and metabolism (animals trained $1 \mathrm{~h}$ /day for six weeks at moderate intensity). During exercise bouts, animals swam with an extra load equivalent to $5.5 \%$ of their total body weight attached to their tail to enhance aerobic training. Physical exercise can improve the macrophage-mediated immune response to pathogen attack (including antigen presentation) in animals with metabolic syndrome. ${ }^{17}$ The above-mentioned studies, however, measured macrophage function 1 and $72 \mathrm{~h}$ after exercise, respectively, and neutrophil phagocytosis was not accessed. These exercise training-induced changes in the immune response can be influenced by multiple factors, including mode, duration and intensity of exercise; they also vary according to the time since the last exercise session. ${ }^{18}$

Considering that the observed differences between study protocols are determinants for immune responses to exercise, the phagocytic response to prolonged exercise is not completely understood, especially for cases in which an adaptation period and different intensities are added. Given that exercise relies on the interplay between both intensity and duration, an experimental design that incorporates both of these variables would increase our understanding of this immunological response. We hypothesized that after adaptation to exercise training, a decrease in the stressor effect of exhausting exercise would occur. Therefore, the purpose of this investigation was to analyze the effects of exhausting long-duration physical exercise of different durations and intensities on the number and phagocytic capacity of macrophages and neutrophils in rats.

\section{MATERIALS AND METHODS}

\section{Animals}

Two-month-old male Wistar rats (Rattus novergicus var. albinus, Rodentia, Mammalia) with a mean weight of $200 \mathrm{~g}$ were used. The animals had free access to water and chow and were kept in collective cages (three rats per cage) at a constant temperature of $23( \pm 2){ }^{\circ} \mathrm{C}$ and a cycle of $12 \mathrm{~h}$ of light and $12 \mathrm{~h}$ of dark (lights on from 06:00 $\mathrm{h}$ to 18:00 h). This research was approved by the Federal University of São Carlos Committee of Experimental Animals.

\section{Experimental Groups}

The animals were divided into two groups: untreated rats (macrophage study) and oyster glycogen-treated rats (neutrophil study). In each group, five subgroups were formed (10 rats per group): unexercised controls, an unadapted lowintensity exercise group, an unadapted moderate-intensity exercise group, a preadapted low-intensity exercise group and a preadapted moderate-intensity group. The animals in the exercised groups performed swimming until exhaustion, and the animals in the preadaptation groups were submitted to four adaptation sessions lasting 5, 15, 30 or 45 min before the exhaustion session. ${ }^{19}$ The rats in the neutrophil study were treated with oyster glycogen approximately $4 \mathrm{~h}$ before exercise. Each animal received an intraperitoneal injection of $10 \mathrm{ml}$ of type II oyster glycogen $(2 \%)$ in PBS to induce the migration of circulating neutrophils to the peritoneal cavity. ${ }^{20}$

\section{Physical Exercise}

The physical exercise model chosen was swimming in a tank with a water temperature of $30 \pm 2{ }^{\circ} \mathrm{C}$ during the afternoon. The unadapted groups were submitted to physical exercise at low or moderate intensity only once (EXAGL and EXAGM, respectively). The animals in the preadapted groups were submitted to four days of adaptation with low- and moderate-intensity exercise (EXADL and EXADM, respectively). The groups exercised at lowintensity were given no additional load. Conversely, the groups submitted to moderate-intensity exercise had a load of $5 \%$ of their body weight placed on their backs, which corresponded to an intensity below the point of inflection of the lactate threshold curve. ${ }^{21,22}$ The collection of cells was performed immediately after the exercise.

\section{Leukometry and Differential Leukogram}

Total leukocyte counts were performed with Turkey liquid and counting in a Neubauer chamber. Differential leukocyte counts were accomplished with an optical microscope using a blood smear colored with MayGrünwald and Giemsa (Sigma, St. Louis, MO, USA).

\section{Total Number of Peritoneal Macrophages and Neutrophils}

PBS (10 ml, pH 7.2) was injected into the animal's peritoneum, and the area was massaged. After the sacrifice, the peritoneum was then longitudinally sectioned, and the injected liquid was collected with a Pasteur pipette. The liquid was placed in a glass tube and centrifuged for $1 \mathrm{~min}$ at $2000 \mathrm{rpm}$ until a pellet was formed. The supernatant was discarded, and the pellet was suspended again in $10 \mathrm{ml}$ of PBS (10 $\times$ dilution, tube 1$)$. A $1 \mathrm{ml}$ sample was extracted from tube 1 and deposited in another tube with $9 \mathrm{ml}$ of PBS $(10 \times$ dilution, tube 2). After this step, $100 \mathrm{ml}$ of the sample in tube 2 was collected and placed in a plastic tube. Then, $100 \mathrm{ml}$ of tryplan blue (Sigma, St. Louis, MO, USA) was added to the plastic tube, and the contents were homogenized with a pipette ( $2 \times$ dilution). The Neubauer chamber was filled, and the numbers of peritoneal macrophages and neutrophils $(4 \mathrm{~h}$ before counting, the animals received an intraperitoneal injection of $10 \mathrm{ml}$ of a solution of type II oyster glycogen) were determined using a microscope. The results are reported as number of cellsN $\times 10^{6}$ [number found at the sum of the four quadrants, 4 (number of quadrants) $\times 50$ $($ dilution $=10 \times 10 \times 2)=$ number $\times 10^{4}$ ].

\section{Phagocytic Capacity of Peritoneal Macrophages and Neutrophils}

Macrophages or neutrophils were incubated with $10 \mathrm{ml}$ of PBS containing zymosan (zymosan: $50 \mu \mathrm{l}$, Sigma, St. Louis, 
MO USA) for $30 \mathrm{~min}$ at $37{ }^{\circ} \mathrm{C}$. After incubation, a $100 \mu \mathrm{l}$ aliquot was extracted and deposited in a plastic assay tube to which $100 \mu \mathrm{l}$ of tryplan blue was added. The Neubauer chamber was filled, and 100 cells (macrophages) were identified. The number of cells among the 100 identified macrophages that had incorporated zymosan was determined; the macrophages were only considered valid if at least three zymozan particles had been incorporated. Therefore, the counting defined the percentage of peritoneal macrophages that had incorporated at least three particles of zymosan, according to the methodological descriptions provided by Curi et al. ${ }^{20}$

\section{Statistical Analysis}

The normality of distribution of all parameters was checked with the Kolmogorov-Smirnov test. The statistical analysis was performed using an ANOVA ( $p \leq 0.05)$. We compared the exercise groups with the control group, the exercise groups given the same duration of exercise to each other and the exercise groups with different protocols. When a significant value was observed, Tukey's post-hoc test was used. The results are expressed as the mean \pm standard error of the mean.

\section{RESULTS}

In the macrophage study, the mean time of swimming until exhaustion was $425.0 \pm 3.0 \mathrm{~min}$. In the study of neutrophils, the swim time was $35.0 \pm 4.0 \mathrm{~min}$.

\subsection{Leukometry and Differential Leukogram}

When compared to the control groups, significant leukocytosis was observed in the unadapted acutely exercised and preadapted groups following exercise to exhaustion at both low and moderate intensities for both protocols ( $\mathrm{P} \leq 0.05$, see Figure 1$)$. For the neutrophil groups, a general increase in the percentage of monocytes and neutrophils in the unadapted groups at low and moderate intensities was observed in the differential leukogram. In addition, a decrease in the percentage of neutrophils in the preadapted groups at low- and moderate-intensity exercise was observed. These effects were not observed in the macrophage study groups.

\section{Total Number and Phagocytic Capacity of Peritoneal Macrophages and Neutrophils}

Macrophage study: There was a significant decrease in the total number of peritoneal macrophages in the unadapted low-intensity group as compared to the control group. Conversely, in the preadapted low-intensity group, the total number of peritoneal macrophages increased, with no changes in the moderate-intensity groups. A significant increase was observed in macrophage phagocytosis in all exercised rats in the macrophage study (Figure 2).

Neutrophil study: The total number of peritoneal neutrophils significantly decreased in the unadapted and preadapted low-intensity groups and increased in the unadapted and preadapted moderate-intensity groups. A significant decrease was observed in neutrophil phagocytosis in the unadapted moderate-intensity group; however, an increase in the preadapted moderate-intensity group was observed, with no changes in the low-intensity groups (Figure 2).

\section{DISCUSSION}

Leukocytosis associated with exhausting long-duration exercise is well described in the literature and was first reported in 1902. ${ }^{23}$ The increase in immune cell count may be caused by a combination of effects of glucocorticoids and catecholamines or merely a redistribution phenomenon. ${ }^{24,25}$ In this study, leukocytosis was observed in both studies regarding macrophages and neutrophils at low and moderate exercise intensities (Figures $1 \mathrm{~A}$ and B).

Monocytes are precursors of macrophages in circulation, and they can be found in free or marginated forms. ${ }^{26}$ In response to acute exercise, monocyte numbers increase as a result of catecholamine-mediated demargination. ${ }^{1,27}$ Exhausting exercise reduces the number of peritoneal macrophages in rats, and the magnitude of this effect is correlated with serum corticosterone concentration. There appears to be an "optimal" physiologic concentration at which glucocorticoids can stimulate macrophages, which is consistent with the idea that low glucocorticoid levels can enhance immunity instead of suppressing it. ${ }^{2,28,29}$ In this study, we observed a tendency for monocytosis in the exercise groups (Figure 1A). We also observed a decrease in the total count of peritoneal macrophages in the unadapted animals submitted to low-intensity exercise and an increase in the animals preadapted to the same exercise regimen (Figure 2A). One potential explanation for this finding could be that muscular training may induce physiological stress during acute exercise, resulting in an increase in the total number of peritoneal macrophages (Figure 2A).

Physical exercise increases the plasmatic concentration of many substances, including glucocorticoids, catecholamines, prolactin (PRL), thyroid hormones and $\beta$-endorphins. ${ }^{30,31}$ All of these substances can enhance the phagocytic capacity of macrophages. ${ }^{1}$ According to Sanders, ${ }^{32}$ most studies addressing the direct effects of catecholamines and the sympathetic nervous system on immunity have examined their effects on adaptive immunity; however, little is known about adrenergic effects on innate immunity. It has been observed that moderate treadmill training upregulates stimulant-induced production of nitric oxide (NO) and cytokines essential for antimicrobial defense in cells of the monocyte/macrophage lineage, suggesting a reduced risk of infection. ${ }^{11}$

Another possible mechanism occurs via mitogen-activated protein kinase (MAPK), which mediates the production of proinflammatory cytokines, whereas MAPK phosphatase (MKP)-1 plays an essential role in intracellular homeostasis by negatively regulating macrophage MAPK activation. Exercise training upregulates basal macrophage MKP-1 expression, accelerates LPS-evoked MKP-1 upregulation, and impacts LPS-evoked immune responses in mice. ${ }^{14}$ Unfortunately, as a limitation of the present study, hormones and intracellular pathways were not studied; however, there was an increase in the phagocytic capacity of macrophages in all of the exercised groups (macrophage study) (Figure 2A). Similarly, another study reported that phagocytic capacity and anion superoxide production by peritoneal macrophages were significantly increased in exercise-trained mice following 12 weeks of moderate exercise training (15-120 $\mathrm{min}$ of treadmill running at a speed of $13 \mathrm{~m} / \mathrm{min}$ for 5 days/week). ${ }^{15}$ Comparisons between our results and those of other studies, however, are difficult to interpret because physiological responses 

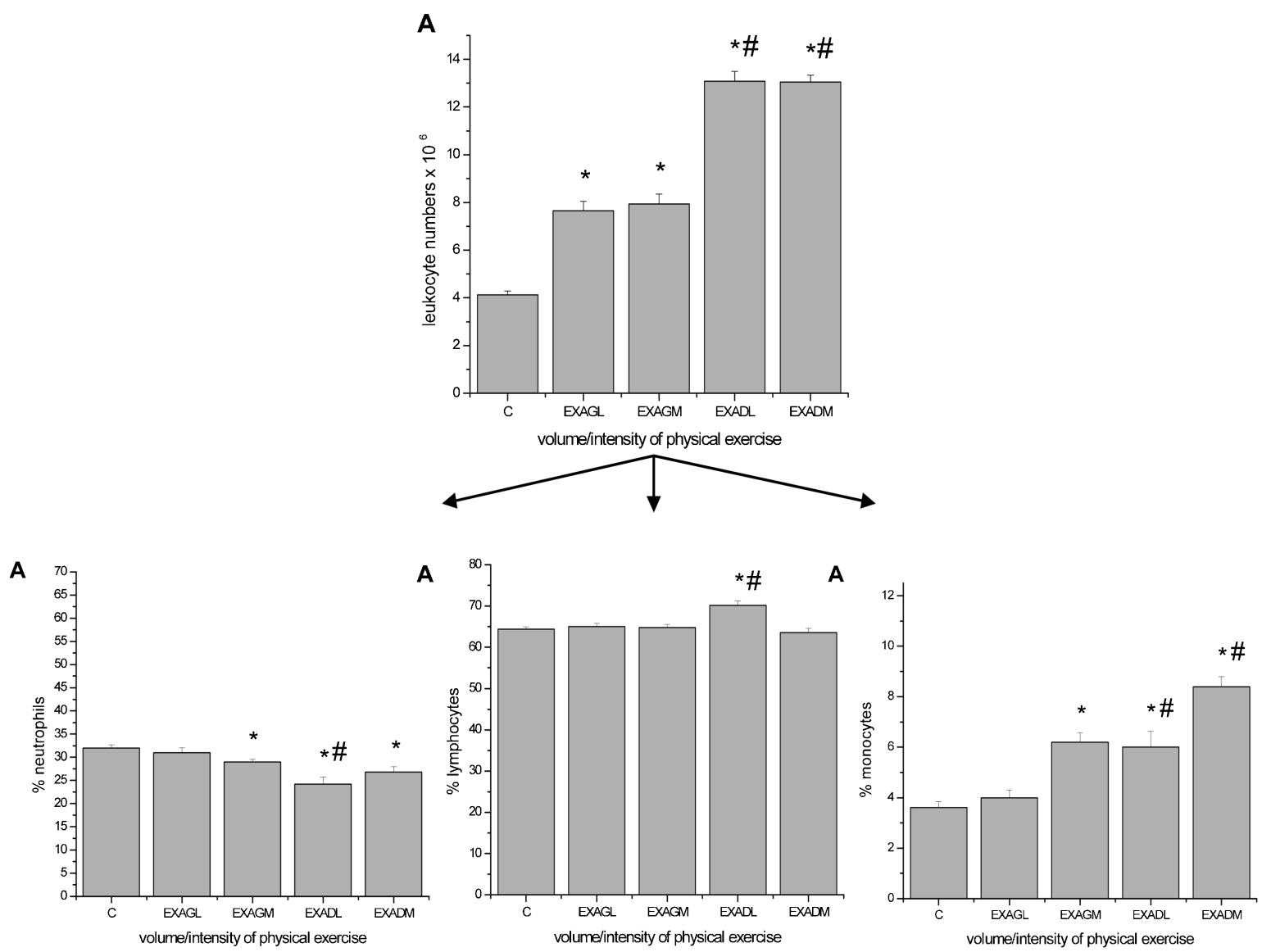

B
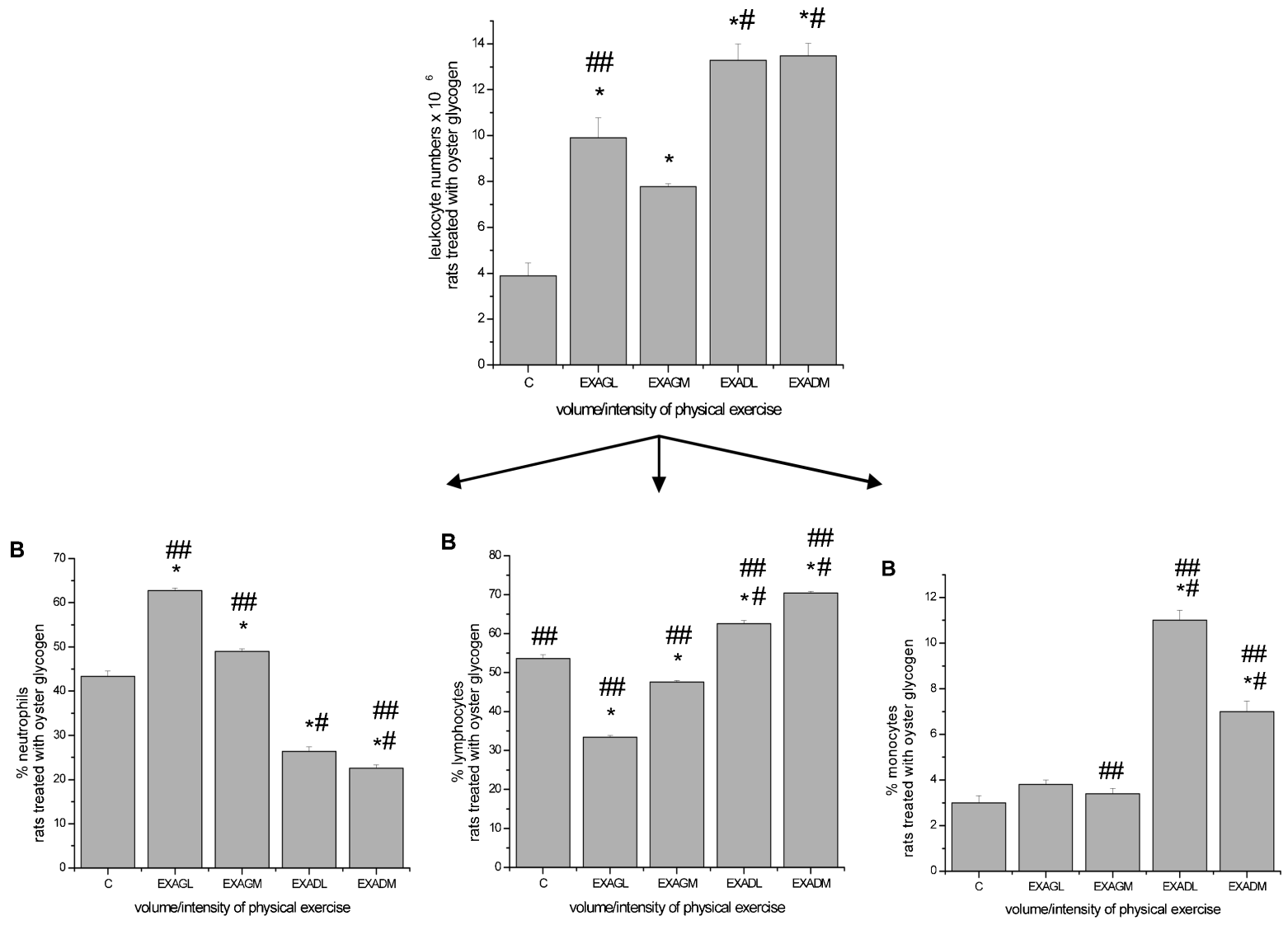
A

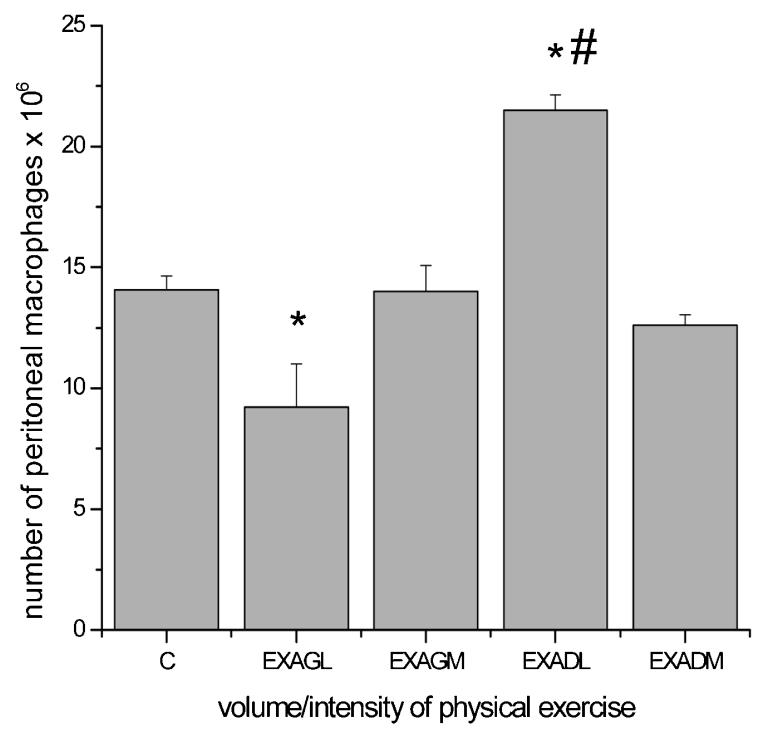

B

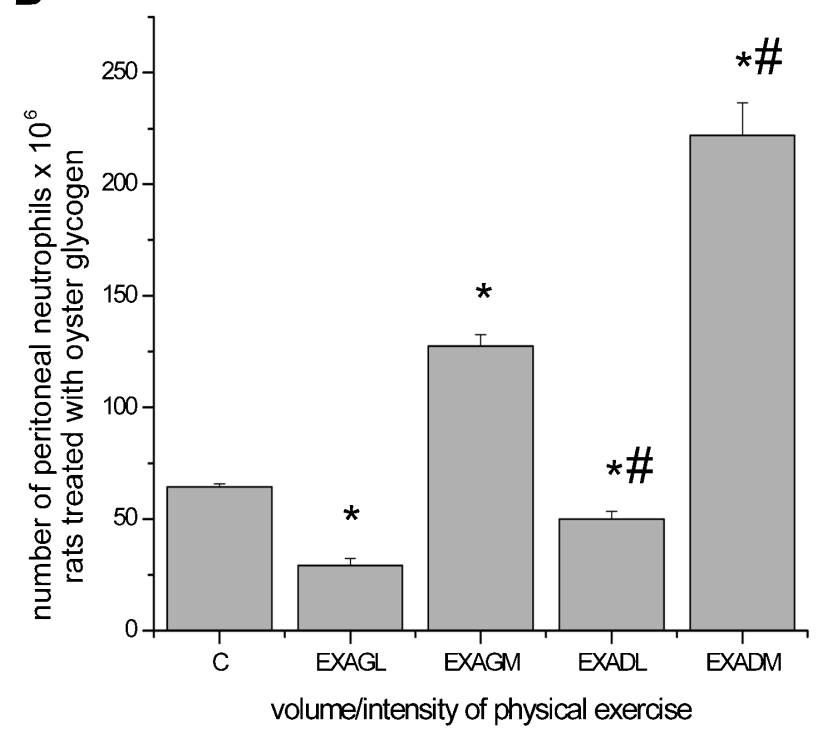

A

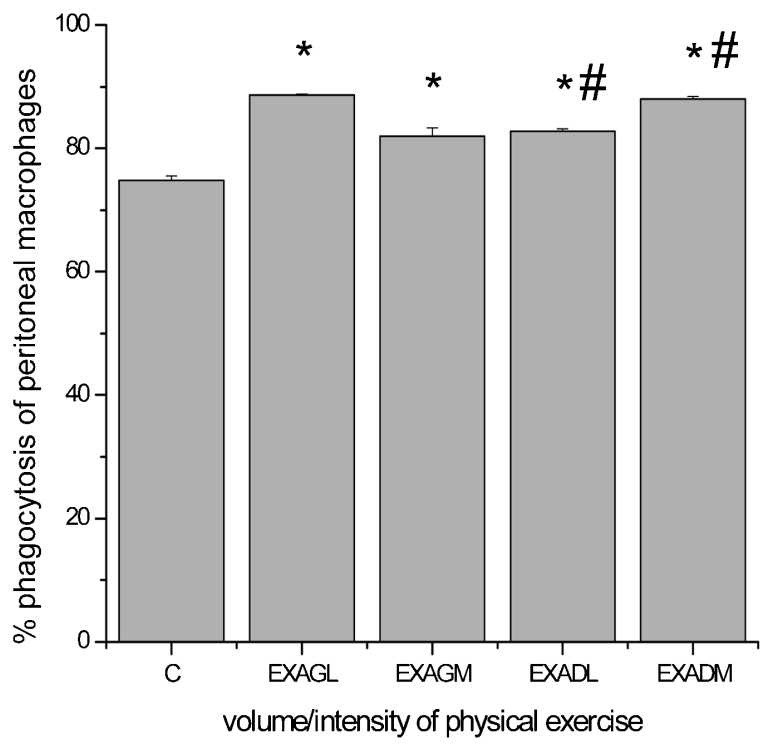

B ${ }^{100} 7$

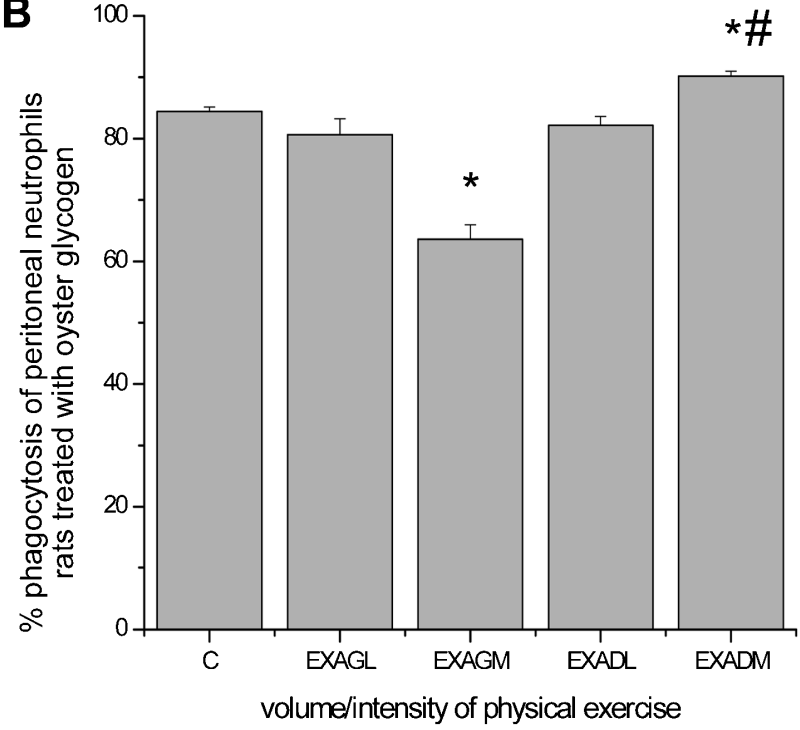

Figure 2 - Total number and phagocytic capacity of peritoneal macrophages and neutrophils. A, macrophage study. B, neutrophil study. The results are expressed as the average number of peritoneal macrophages and neutrophils and the percentage of phagocytosis \pm standard error of the average. ${ }^{*} p \leq 0.05$ for exercise groups submitted to low- and moderate-intensity exercise (unadapted low intensity = EXAGL; moderate intensity = EXAGM and corresponding preadapted groups, EXADL and EXADM) as compared to the control group (C); \# $\mathrm{p} \leq 0.05$ for comparisons made among the groups.

depend on variables of physical exercise, such as intensity, duration and frequency as well as exercise type. In addition, in other studies, the time at which the immune response is analyzed following exercise is widely variable.

Catecholamines have been shown to modulate neutrophil cell levels. ${ }^{25}$ An increase in catecholamines depends on the exercise intensity, duration and adaptation. Moreover, catecholamines promote acute exercise effects on neutrophils, whereas cortisol may be responsible for the maintenance of neutrocytosis after long-duration exercise. ${ }^{33}$ In this study, we observed neutrocytosis in unadapted animals submitted to an exercise session at low and moderate intensities (neutrophil study) and neutropenia in animals that were preadapted to the same exercise regimens

Figure 1 - Leukometry and differential leukogram with the percentage of circulating neutrophils, lymphocytes and monocytes. A, macrophage study. B, neutrophil study. The results are expressed as the average number of leukocytes and the percentage of cells \pm standard error of the average. *, $\mathrm{p} \leq 0.05$ for exercise groups submitted to low- and moderate-intensity exercise (unadapted low intensity = EXAGL; moderate intensity = EXAGM and corresponding preadapted groups, EXADL and EXADM) as compared to the control group (C); \# $\mathrm{p} \leq 0.05$ for comparisons among the groups; \#\# $\mathrm{p} \leq 0.05$ for comparisons made between the macrophage and neutrophil study groups. 
(Figure 1B). Regarding the total number of peritoneal neutrophils, a decrease occurred in the unadapted and preadapted groups submitted to low-intensity exercise (neutrophil study), and an increase was noted in the groups that exercised at moderate intensity (Figure 2B). It is possible that the magnitude of the physiological stress caused a redistribution of neutrophils during exercise and adaptation, as was observed in our preadapted groups submitted to both exercise intensities (neutrophil study) (Figure 2B). An increase in the total number of peritoneal neutrophils in the groups that completed moderate-intensity exercise (unadapted and preadapted) was observed, and the migration of neutrophils to the peritoneal cavity increased proportionally with the intensity of exercise, consistent with previous findings. ${ }^{33}$

Neutrophil phagocytic capacity is stimulated mainly by glucocorticoids and catecholamines, as neutrophils have receptors for these substances. ${ }^{1}$ Adrenaline is a potent modulator of the immune system, and its level increases with exercise intensity. This hormone is capable of reducing the phagocytic capacity of peritoneal neutrophils in vitro. ${ }^{34}$ In addition, noradrenaline seems to stimulate neutrophil functionality. A study with rats subjected to sympathetic inhibition demonstrated a reduction in chemotaxis and phagocytosis as compared to control rats. ${ }^{6,7}$ In the context of our investigation, a reduction was observed in peritoneal neutrophil phagocytic capacity in the group submitted to a moderate-intensity exercise session, whereas an increase was observed in the preadapted group subjected to the same level of exercise (neutrophil study) (Figure 2B). The increase associated with the preadapted group submitted to moderate-intensity exercise may have resulted from a decreased physiological stress response because of the adaptation to physical exercise (Figure 2B). Future investigations directed at testing this hypothesis are, therefore, warranted. The major limitation of our data is that hormone levels were not measured. Despite this limitation, the current data demonstrate important novel findings regarding exercise intensity and adaptation prior to exhaustive endurance exercise.

In general, exhausting long-duration physical exercise was found to reduce the total number of peritoneal macrophages following low-intensity exercise; however, this variable was increased in a separate group of animals submitted to four adaptation sessions. The phagocytic capacity of all groups, however, increased. Conversely, the total number of peritoneal neutrophils decreased in the groups submitted to low-intensity exercise and increased in the groups performing moderate-intensity exercise. In addition, the phagocytic capacity decreased in the sedentary group following acute moderate-intensity exercise, whereas an increase was observed in the preadapted groups.

It is possible that the adaptive response to exercise training resulted in a decrease in the stressor effect of exhausting exercise. In sedentary rats, neutrophils and macrophages respond differently to the stress of exercise. In this sense, adaptation sessions may be important for individuals who are beginning an exercise-training program, to thus avoid excessive physiological stress on the immune system.

\section{ACKNOWLEDGEMENTS}

We thank FAPESP, PIBIC/CNPq, FAPIC/UNIMEP, FAP/UNIMEP and CAPES/PROSUP for financial support and all of the individuals that participated direct or indirectly in this research.

\section{REFERENCES}

1. Ortega E. Neuroendocrine mediators in the modulation of phagocytosis by exercise: physiological implications. Exerc Immunol Rev. 2003;9:7093.

2. Ortega E. Influence of exercise on phagocytosis. Int J Sports Med. 1994;15:S172-S178, doi: 10.1055/s-2007-1021133.

3. Nieman DC, Pedersen BK. Exercise and immune function. Recent developments. Sports Med. 1999;27:73-80, doi: 10.2165/00007256199927020-00001

4. Munk A, Guyre PM. Glucocorticoids and imune function. In: Ader R, Felten DL, Cohen N. Psychoneuroimmunology. New York: Academic Press. 1991.

5. Weigent DA, Blalock JE. Associations between the neuroendocrine and immune systems. J Leukoc Biol 1995;58:137-50.

6. Carter L, Ferrari JK, Davison JS, Befus D. Inhibition of neutrophil chemotaxis and activation following decentralization of the superior cervical ganglia. J Leukoc Biol. 1992;51:597-602.

7. Nagatomi R, Kaifu T, Okutsu M, Zhang X, Kanemi O, Ohmori H. Modulation of the immune system by the autonomic nervous system and its implication in immunological changes after training. Exerc Immunol Rev. 2000;6:54-74.

8. Rincón EO, Marchena JM, García JJ, Schmidt A, Schulz T, Malpica I. et al. Phagocytic function in cyclists: correlation with catecholamines and cortisol. J Appl Physiol. 2001;91:1067-72.

9. Bernton EW, Bryant HU, Holaday JW. Prolactin and immune function. In: Ader R, Felten DL, Cohen N. Psychoneuroimmunology. New York: Academic Press, 1991.

10. Kelly KW. Growth hormone in Immunobiology. In: Ader R, Felten DL, Cohen N. Psychoneuroimmunology. New York: Academic Press, 1991.

11. Kizaki T, Takemasa T, Sakurai T, Izawa T, Hanawa T, Kamiya S, et al. Adaptation of macrophages to exercise training improves innate immunity. Biochem Bioph Res Com. 2008;372:152-6, doi: 10.1016/j.bbrc. 2008.05.005.

12. Batista ML Jr, Santos RV, Oliveira EM, Seelaender MC, Costa Rosa LF. Endurance training restores peritoneal macrophage function in post-MI congestive heart failure rats. J Appl Physiol. 2007;102:2033-9, doi: 10. 1152/japplphysiol.00871.2006.

13. Vitorino DC, Buzzachera CF, Curi R, Fernandes LC. Effect of chronic supplementation with shark liver oil on immune responses of exercisetrained rats. Eur J Appl Physiol. 2010;108:1225-32, doi: 10.1007/s00421009-1267-3.

14. Chen MF, Chen HI, Jen CJ. Exercise Training Upregulates Macrophage MKP-1 and Impacts Immune Responses in Mice. Med Sci Sports Exerc. 2010;16: in press.

15. Sugiura H, Nishida H, Inaba R, Mirbod SM, Iwata H. Effects of different durations of exercise on macrophage functions in mice. J Appl Physiol. 2001;90:789-94, doi: 10.1063/1.1381000.

16. Dos Santos RVT, Caperuto EC, de Mello MT, Costa Rosa, LFBP. Effect of exercise on glutamine metabolism in macrophages of trained rats. Eur J Appl Physiol. 2009;107:309-15, doi: 10.1007/s00421-009-1130-6.

17. Martin-Cordero L, Garcia JJ, Giraldo E, De la Fuente M, Manso R, Ortega E. Influence of exercise on the circulating levels and macrophage production of IL-1 $\beta$ and IFN $\gamma$ affected by metabolic syndrome: an obese Zucker rat experimental animal model. Eur J Appl Physiol 2009;107:53543, doi: 10.1007/s00421-009-1140-4.

18. Pedersen BK, Toft AD. Effects of exercise on lymphocytes and cytokines. Br J Sports Med. 2000;34:246-51, doi: 10.1136/bjsm.34.4.246.

19. Sampaio-Barros MM, Farias-Silva E, Grassi-Kassisse DM, SpadariBratfisch RC. Effect of swimming session duration and repetition on metabolic markers in rats. Stress. 2003;6:127-32, doi: 10.1080/ 1025389031000110169.

20. Curi TC, Melo MP, Azevedo RB, Curi R. Glutamine utilization by rat neutrophils. Biochem Soc Trans. 1997;25:249S.

21. Gobatto CA, Mello MA, Sibuya CY, Azevedo JR, Santos LA, Kokubun E. Maximal lactate steady state in rats submitted to swimming exercise. Comp. Biochem Physiol A Mol Integr Physiol. 2001;130:21-7, doi: 10. 1016/S1095-6433(01)00362-2.

22. Voltarelli FA, Gobatto CA, Mello MA. Determination of anaerobic threshold in rats using the lactate minimum test. Braz. J Med Biol Res. 2002;35:1389-94, doi: 10.1590/S0100-879X2002001100018.

23. Larrabee RC. Leucocytosis after violent exercise. J Med Res 1902;7:76-82.

24. Barriga C, Pedrera MI, Maynar M, Maynar J, Ortega E. Effect of submaximal physical exercise performed by sedentary men and women on some parameters of the immune system. Rev Esp Fisiol. 1993;49:79-85.

25. Brenner I, Shek PN, Zamecnik J, Shephard RJ. Stress hormones and the immunological responses to heat and exercise. Int J Sports Med. 1998;19:130-43, doi: 10.1055/s-2007-971895.

26. van Furth R, Sluiter W. Distribution of blood monocytes between a marginating and a circulating pool. J Exp Med. 1986;163:474-9, doi: 10. 1084/jem.163.2.474.

27. Woods JA, Davis JM, Smith JA, Nieman DC. Exercise and cellular innate immune function. Med Sci Sports Exerc. 1999;31:57-66, doi: 10.1097/ 00005768-199901000-00011. 
28. Ortega E, Collazos ME, Barriga C, De la Fuente M. Stimulation of the phagocytic function in guinea pig peritoneal macrophages by physical activity stress. Eur J Appl Physiol Occup Physiol. 1992;64:323-7, doi: 10.1007/BF00636219.

29. Forner MA, Barriga C, Rodriguez AB, Ortega E. A study of the role of corticosterone as a mediator in exercise-induced stimulation of murine macrophage phagocytosis. J Physiol. 1995;488(Pt3):789-94.

30. Khansari DN, Murgo AJ, Faith RE. Effects of stress on the immune system. Immunol Today. 1990;11:170-5, doi: 10.1016/0167-5699(90)90069-L.

31. Simon HB. Exercise and human immune function. In: Ader R, Felten DL, Cohen N. Psychoneuroimmunology. New York: Academic Press, 1991.
32. Sanders VM. Interdisciplinary research: noradrenergic regulation of adaptive immunity, Brain Behav Immun. 2006;20:1-8, doi: 10.1016/j.bbi. 2005.07.004.

33. Peake JM. Exercise-induced alterations in neutrophil degranulation and respiratory burst activity: possible mechanisms of action. Exerc Immunol Rev 2002;8:49-100.

34. Garcia C, Pithon-Curi TC, de Lourdes Firmano M, Pires de Melo M, Newsholme P, Curi R. Effects of adrenaline on glucose and glutamine metabolism and superoxide production by rat neutrophils. Clin Sci. (Lond) 1999;96:549-55, doi: 10.1042/CS19980340. 